Article

\title{
Discovery of Potent Carbonic Anhydrase and Acetylcholinesterase Inhibitors: 2-Aminoindan $\beta$-Lactam Derivatives
}

\author{
Hayriye Genç ${ }^{1}$, Ramazan Kalin ${ }^{2}$, Zeynep Köksal ${ }^{3}$, Nastaran Sadeghian ${ }^{4}$, Umit M. Kocyigit ${ }^{5}$, \\ Mustafa Zengin ${ }^{1}$, İlhami Gülçin ${ }^{4,6, *}$ and Hasan Özdemir 4 \\ 1 Department of Chemistry, Faculty of Arts and Sciences, Sakarya University, Sakarya 54050, Turkey; \\ hayriyegenc@sakarya.edu.tr (H.G.); mzengin@sakarya.edu.tr (M.Z.) \\ 2 Department of Basic Science, Faculty of Science, Erzurum Technical University, Erzurum 25700, Turkey; \\ rkalin@atauni.edu.tr \\ 3 Department of Chemistry, Faculty of Sciences, İstanbul Medeniyet University, İstanbul 34730, Turkey; \\ zkoksal@atauni.edu.tr \\ 4 Department of Chemistry, Faculty of Science, Atatürk University, Erzurum 25240, Turkey; \\ parham_taslimi_un@yahoo.com (N.S.); hozdemir@atauni.edu.tr (H.Ö.) \\ 5 Vocational School of Health Services, Cumhuriyet University, Sivas 58140, Turkey; \\ ukocyigit@cumhuriyet.edu.tr \\ 6 Department of Zoology, College of Science, King Saud University, Riyadh 11451, Saudi Arabia \\ * Correspondence: igulcin@atauni.edu.tr; Tel.: +90-442-231-4375; Fax: +90-442-231-4109
}

Academic Editor: Mateus Webba da Silva

Received: 17 July 2016; Accepted: 10 October 2016; Published: 20 October 2016

\begin{abstract}
Lactams are pharmacologically important compounds because of their various biological uses, including antibiotic and so on. $\beta$-Lactams were synthesized from benzylidene-inden derivatives and acetoxyacetyl chloride. The inhibitory effect of these compounds was examined for human carbonic anhydrase I and II (hCA I, and II) and acetylcholinesterase (AChE). The results reveal that $\beta$-lactams are inhibitors of hCA I, II and AChE. The $K_{i}$ values of $\beta$-lactams $(\mathbf{2 a}-\mathbf{k})$ were $0.44-6.29 \mathrm{nM}$ against hCA I, 0.93-8.34 nM against hCA II, and 0.25-1.13 nM against AChE. Our findings indicate that $\beta$-lactams (2a-k) inhibit both carbonic anhydrases (CA) isoenzymes and AChE at low nanomolar concentrations.
\end{abstract}

Keywords: carbonic anhydrase; acetylcholinesterase; $\beta$-lactam; 2-azetidinone; enzyme inhibition; enzyme purification

\section{Introduction}

The $\beta$-lactams can be classified into several groups according to their structural characteristics, but their unique structural feature is the presence of the four-membered $\beta$-lactam (2-azetidinone) ring [1]. 2-Azetidinone containing antibiotics are known as $\beta$-lactam antibiotics and they are the most widely employed family of antibacterial agents [2]. Moreover, they have been reported as having antibacterial, anticancer, and antiviral activity, and an enzyme inhibition effect [3-5]. The investigation of the chemistry and biology of $\beta$-lactam continue to appeal to synthetic and medicinal organic chemists [6]. They have also been used for the preparation of different heterocyclic compounds of biological significance [7].

Carbonic anhydrases (CAs, E.C. 4.2.1.1) catalyse a very simple but physiologically essential reaction in all life kingdoms: the hydration of carbon dioxide $\left(\mathrm{CO}_{2}\right)$ and water $\left(\mathrm{H}_{2} \mathrm{O}\right)$ to bicarbonate $\left(\mathrm{HCO}_{3}{ }^{-}\right)$and protons $\left(\mathrm{H}^{+}\right)$with a high efficiency [8-11]. CAs are metalloenzymes that participate in the control of $\mathrm{pH}$ in the body, are encoded by six different independent gene families $\left(\alpha-, \beta-, \gamma_{-}, \delta_{-}, \zeta-\right.$, 
and $\eta-C A$ ) and are found in eukaryotic and prokaryotic cells [12-15]. They catalyse reversible $\mathrm{CO}_{2}$ hydration, which are essential important physiologic processes in all life kingdoms [16-18].

$$
\mathrm{CO}_{2}+\mathrm{H}_{2} \mathrm{O} \stackrel{\mathrm{CA}}{\Leftrightarrow} \mathrm{H}_{2} \mathrm{CO}_{3} \Leftrightarrow \mathrm{HCO}_{3}^{-}+\mathrm{H}^{+}
$$

Living organisms possess sixteen CA isoenzymes, which differ in their subcellular localization and catalytic activity [19-22]. They were found in various organs and tissues with different kinetic and molecular properties, expression levels, and oligomeric rearrangements as well as various abilities to respond to different inhibitory classes [23-25]. These enzymes play a very important role in different tissues [26,27]. CA I, II, III, VII, and XIII isoenzymes are cytosolic, CA IV, IX, XII, and CA XIV are bound to membranes, CA VA-VB are mitochondrial, and CA VI are in the milk and saliva [28,29]. The erythrocytes contain CA I, and II at high concentrations. The CA inhibitors (CAIs) are used as a class of pharmaceuticals, which used as diuretics, anti-glaucoma agents. Also, they used for treatment of gastric and duodenal ulcers, neurological disorders, and osteoporosis [30-32]. Up until now, the inhibitory effects against different $C$ As types have been investigated for different sulphonamides derivatives, heavy metal ions, phenols, antibiotics, and various drugs [33-35]. $\beta$-Lactams are widely used in the treatment of many diseases. However, there is no detailed study on erythrocytes hCA I, and II isoenzymes of $\beta$-lactams (2a-k).

Acetylcholinesterase (AChE) is a crucial enzyme used for transmission control between neurons [36] when the process is either mediated or modulated by the neurotransmitter acetylcholine (ACh). ACh is released by the axon terminal or varicosities of the transmitter neuron into the extracellular space to interact with the receptors of the other neuron. AChE hydrolyses ACh to choline and acetate $[37,38]$. If $\mathrm{AChE}$ is inhibited in the central nervous system, then the concentration of ACh increases in the synaptic cleft, leading to cholinergic crisis, which affords several dangerous effects, such as convulsions and respiratory problems, which could lead to death. AChE inhibitors (AChEIs) have medical applications and are particularly important for the symptomatic treatment of Alzheimer's disease (AD) to enhance central cholinergic transmission [39]. AD is a fatal and chronic neurodegenerative disease that usually starts slowly and gets worse over time [19]. From this perspective, there is a great need for improved AChEIs that show low toxicity, good brain penetration, and high bioavailability. The use of AChEIs to block the cholinergic degradation of acetylcholine (Ach) is therefore considered to be a promising approach for the treatment of $\mathrm{AD}[40,41]$.

In the present study, we investigated the inhibition profile of a series of $\beta$-lactam derivatives (2a-k) against CA I, and II isoforms from human erythrocytes and AChE enzyme.

\section{Results and Discussion}

$\beta$-Lactam derivatives are drugs that protect against many different gram positive-negative and anaerobic organisms. They are perhaps among the best-studied and most widely used antibiotics in the world [42]. The general synthesis route of the novel $\beta$-lactam derivatives $(\mathbf{2} \mathbf{a}-\mathbf{k})$ is shown in Figure 1.

Herein, $\beta$-lactam derivatives (2a-k) were prepared from benzylidene-inden derivatives and ketene and characterized by NMR and MS. We assumed that the synthesized compounds were in cis form based on the literature [43-45]. The chemical structures of $\mathbf{2} \mathbf{a}-\mathbf{k}$ are given in Figure 2 . The in vitro inhibitory effects of compounds $\mathbf{2} \mathbf{a}-\mathbf{k}$ were also examined for purified hCA I, and II isoenzymes activities using the esterase activity method. $\beta$-Lactam derivatives inhibit growth of sensitive bacteria by inactivating enzymes in the cell membrane. $\beta$-Lactam derivatives were synthesized and evaluated as inhibitors of protease, elastase, and the cysteine protease papain. Some drug molecules are enzyme inhibitors, so their discovery is important in biochemistry research [46]. Inhibitors of CA have several medical applications, such as in treating glaucoma disease, high blood pressure, and the neurological disorders epilepsy and Alzheimer's disease. Some research groups are currently working on the synthesis of new inhibitors of the carbonic anhydrase family for the treatment of some diseases [47-49]. 


\begin{tabular}{cc}
\hline Compounds & $\mathbf{R}$ \\
\hline $\mathbf{a}$ & $-\mathrm{H}$ \\
$\mathbf{b}$ & $-m \mathrm{OMe}$ \\
$\mathbf{c}$ & $-p \mathrm{Me}$ \\
$\mathbf{d}$ & $-m \mathrm{Me}$ \\
$\mathbf{e}$ & $-m \mathrm{Cl}$ \\
$\mathbf{f}$ & $-m, p-\mathrm{diCl}$ \\
$\mathbf{g}$ & $-m \mathrm{Br}$ \\
$\mathbf{h}$ & $-o \mathrm{Br}$ \\
$\mathbf{i}$ & $-p \mathrm{Br}$ \\
$\mathbf{j}$ & $-o \mathrm{NO}_{2}$ \\
$\mathbf{k}$ & $-p \mathrm{~F}$ \\
\hline
\end{tabular}

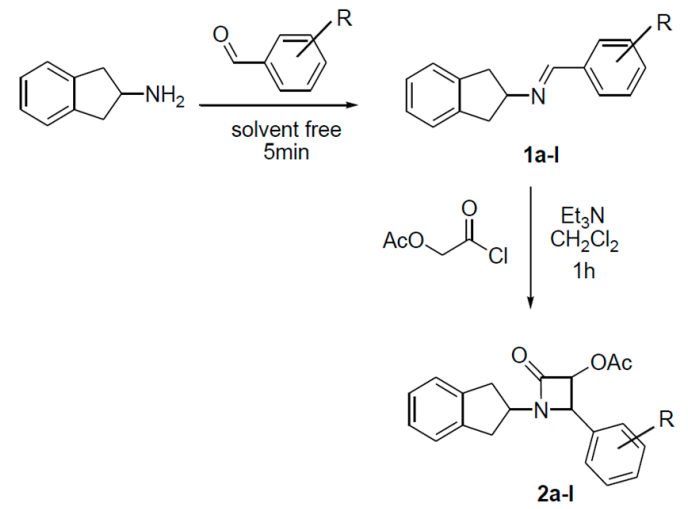

Figure 1. General synthesis route of novel $\beta$-lactam derivatives (2a-k).
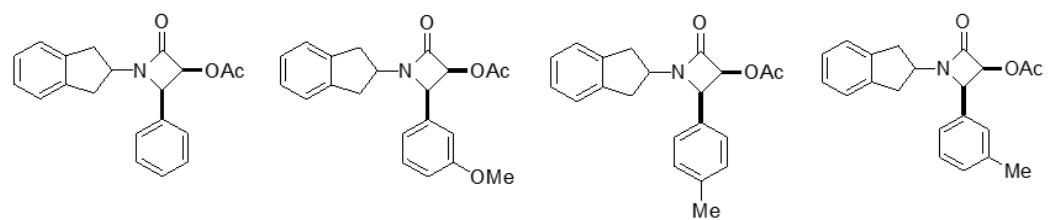

2a

2b

2c

2d
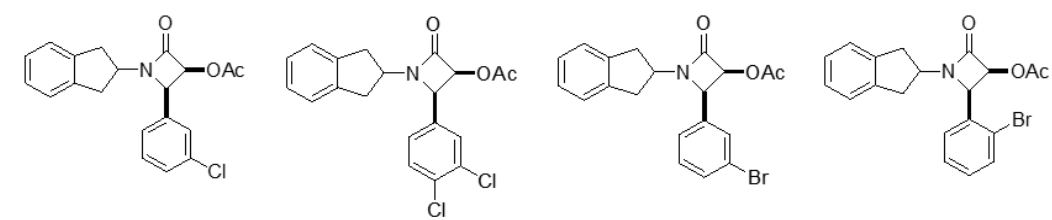

$2 \mathbf{e}$

2f

$2 \mathrm{~g}$

2h

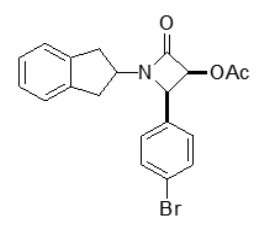<smiles>O=C(O)C(c1ccccc1[N+](=O)[O-])N(Cc1ccccc1)C(=O)[O-]</smiles><smiles>COC(=O)N(Cc1ccccc1)c1ccc(F)cc1</smiles>

$2 \mathbf{j}$

$2 \mathbf{k}$

Figure 2. The chemical structures of the investigated $\beta$-lactam derivatives (2a-k).

Some chemicals at low dosages are effective by altering normal enzyme activity and by inhibiting a specific enzyme [50,51]. It is well known that $\beta$-lactams had inhibition properties on hCA I, and II isoenzymes and are used in therapies [52,53]. The inhibition effects of newly synthesized compounds $\mathbf{2 a}-\mathbf{k}$ were determined for the first time against hCA I, and II. For this purpose, as shown in Table 1, hCA I and II were separately purified from erythrocytes with affinity chromatography. The hCA I was purified 127.9-fold with a specific activity of $1151.4 \mathrm{EU} / \mathrm{mg}$ and overall yield of $63.9 \%$, and the hCA II enzyme was purified 788.9-fold with a specific activity of $7100.0 \mathrm{EU} / \mathrm{mg}$ and overall yield of $56.4 \%$ (Table 1). The purification was monitored by SDS-PAGE. After this process, a single band was observed for each isoenzyme (Figure 3). For the compounds, the inhibitor concentrations causing up to $50 \%$ inhibition ( $\mathrm{IC}_{50}$ values) were determined from the regression analysis graphs. From in vitro studies, it is understood that hCA I, hCA II, and AChE were inhibited by these $\beta$-lactam compounds $\mathbf{2 a}-\mathbf{k}$ (Table 2). The inhibition data of $\beta$-lactam derivatives $\mathbf{2 a - k}$ reported here are shown in Table 2 , and the following comments can be drawn from these data: 
Table 1. Summary of purification procedure for human carbonic anhydrase (hCA) I, and II from fresh human erythrocytes with Sepharose-4B-L-tyrosine-sulphanilamide affinity chromatography.

\begin{tabular}{cccccccc}
\hline Purification Steps & & $\begin{array}{c}\text { Volume } \\
(\mathbf{m L})\end{array}$ & $\begin{array}{c}\text { Total Enzyme } \\
\text { Activity (EU) }\end{array}$ & $\begin{array}{c}\text { Total } \\
\text { Protein } \\
\text { (mg) }\end{array}$ & $\begin{array}{c}\text { Specific } \\
\text { Activity } \\
\text { (EU/mg) }\end{array}$ & $\begin{array}{c}\text { Yield } \\
\text { (\%) }\end{array}$ & $\begin{array}{c}\text { Purification } \\
\text { Fold }\end{array}$ \\
\hline Hemolysate & 50 & 6300 & 700 & 9.0 & 100 & 1 \\
\hline $\begin{array}{c}\text { Sepharose-4B-L-tyrosine- } \\
\text { sulphanilamide affinity } \\
\text { chromatography }\end{array}$ & hCA I & 10 & 4030 & 3.5 & 1151.4 & 63.9 & 127.9 \\
\cline { 2 - 8 } & hCA II & 5 & 3550 & 0.50 & 7100.0 & 56.4 & 788.9 \\
\hline
\end{tabular}

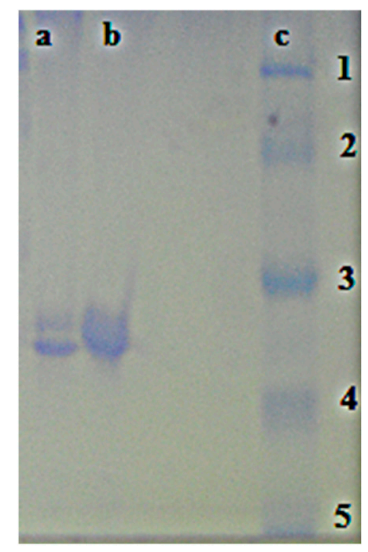

Figure 3. Sodium dodecyl sulphate-polyacrylamide gel electrophoresis bands of carbonic anhydrase I and II isoenzymes and standard proteins. Lanes a: hCA II, b: hCA I, c: standard proteins; standards 1: $116 \mathrm{kDa}$ ( $\beta$-Galactosidase from E. Coli), 2: $97 \mathrm{kDa}$ (Phosphorilase from rabbit muscle), 3: $66 \mathrm{kDa}$ (Bovine serum albumin), 4: $45 \mathrm{kDa}$ (Ovalbumin), 5: $29 \mathrm{kDa}$ (carbonic anhydrase from bovine erythrocyte).

Table 2. Human carbonic anhydrase isoenzymes (hCA I and II) inhibition value with $\beta$-lactam derivatives $\mathbf{2} \mathbf{a}-\mathbf{k}$ by an esterase assay with $p$-nitrophenylacetate (NPA) as a substrate.

\begin{tabular}{|c|c|c|c|c|c|c|c|c|c|}
\hline \multirow{2}{*}{ Compounds } & \multicolumn{6}{|c|}{$\mathrm{IC}_{50}(\mathrm{nM})$} & \multicolumn{3}{|c|}{$K_{i}(\mathrm{nM})$} \\
\hline & hCA I & $R^{2}$ & hCA II & $R^{2}$ & AChE & $R^{2}$ & hCA I & hCA II & AChE \\
\hline $2 a$ & 0.612 & 0.9578 & 5.212 & 0.9099 & 0.885 & 0.9960 & $0.44 \pm 0.115$ & $3.54 \pm 0.405$ & $1.13 \pm 0.472$ \\
\hline $2 b$ & 2.303 & 0.9906 & 6.418 & 0.9009 & 0.913 & 0.9868 & $1.41 \pm 0.547$ & $3.15 \pm 1.139$ & $0.39 \pm 0.069$ \\
\hline $2 c$ & 2.107 & 0.9400 & 3.707 & 0.9012 & 0.783 & 0.9913 & $1.50 \pm 0.657$ & $2.96 \pm 0.157$ & $0.55 \pm 0.136$ \\
\hline $2 d$ & 0.231 & 0.9704 & 4.176 & 0.9029 & 0.634 & 0.9926 & $1.49 \pm 0.290$ & $3.26 \pm 0.708$ & $0.77 \pm 0.041$ \\
\hline $2 e$ & 3.984 & 0.9107 & 5.825 & 0.9136 & 0.668 & 0.9724 & $6.29 \pm 2.068$ & $8.34 \pm 3.530$ & $0.42 \pm 0.020$ \\
\hline $2 f$ & 1.627 & 0.9936 & 5.975 & 0.9230 & 0.602 & 0.9791 & $1.16 \pm 0.514$ & $1.50 \pm 0.421$ & $0.46 \pm 0.045$ \\
\hline $2 \mathrm{~g}$ & 0.652 & 0.9000 & 3.809 & 0.9295 & 0.734 & 0.9803 & $0.35 \pm 0.105$ & $3.39 \pm 1.158$ & $0.44 \pm 0.057$ \\
\hline $2 \mathrm{~h}$ & 2.646 & 0.9264 & 5.212 & 0.9662 & 0.450 & 0.9947 & $0.91 \pm 0.143$ & $2.19 \pm 0.921$ & $0.25 \pm 0.019$ \\
\hline 21 & 2.548 & 0.9359 & 4.030 & 0.9604 & 0.705 & 0.9984 & $0.97 \pm 0.245$ & $0.93 \pm 0.295$ & $0.36 \pm 0.045$ \\
\hline $2 \mathrm{j}$ & 4.814 & 0.9284 & 5.023 & 0.9057 & 0.704 & 0.9746 & $1.09 \pm 0.136$ & $2.88 \pm 1.168$ & $0.56 \pm 0.073$ \\
\hline $2 \mathrm{k}$ & 2.502 & 0.9886 & 6.863 & 0.9132 & 0.859 & 0.9939 & $0.94 \pm 0.430$ & $1.34 \pm 0.539$ & $0.68 \pm 0.117$ \\
\hline $\operatorname{AZA}^{\Psi, *}$ & 101.19 & 0.9509 & 113.75 & 0.9791 & - & - & $170.34 \pm 2.48$ & $115.43 \pm 1.63$ & - \\
\hline TAC $\mathscr{H}$ & - & - & - & - & 4.101 & 0.9951 & - & - & $3.90 \pm 0.792$ \\
\hline
\end{tabular}

${ }^{\Psi}$ Acetazolamide (AZA) was used as a standard inhibitor for both carbonic anhydrase (CA) isoenzymes;

Ho Tacrine (TAC) was used as a standard inhibitor for all AChE; * These values were obtained from [54].

(1) Cytosolic hCA I is expressed in the body and can be found in high concentrations in the blood and gastrointestinal tract. All $\beta$-lactam derivatives $\mathbf{2} \mathbf{a}-\mathbf{k}$ exhibited effective inhibitory activity against the cytosolic isoenzyme hCA I with a $K_{i}$ value of $0.35 \pm 0.105-6.29 \pm 2.068 \mathrm{nM}$ (Table 1). Also, $\beta$-lactam derivative $\mathbf{2 g}$ shown the most powerful CA I inhibition effect with a $K_{i}$ value of $0.35 \pm 0.105 \mathrm{nM}$. On the other hand, we found that acetazolamide (AZA), which is used as a clinical CA inhibitor in the treatment of glaucoma, cystinuria, epilepsy, altitude sickness, periodic paralysis, dural ectasia, idiopathic intracranial hypertension, and central sleep apnoea [52], has a $K_{i}$ value of $170.34 \pm 2.48 \mathrm{nM}$ 
(Table 2). The results clearly show that all $\beta$-lactam derivatives $\mathbf{2 a - k}$ demonstrate more effective hCA inhibitory activity than that of AZA.

(2) With regard to the profiling assay against cytosolic hCA II, $\beta$-lactam derivatives $\mathbf{2 a - k}$ have similar inhibition effects; with $K_{i}$ values ranging from $0.93 \pm 0.295$ through $8.34 \pm 3.530 \mathrm{nM}$. For comparison, AZA, which is used as a clinical CA inhibitor showed a $K_{i}$ value of $115.43 \pm 1.63 \mathrm{nM}$. This result clearly shows that all $\beta$-lactam derivatives $\mathbf{2 a}-\mathbf{k}$ are a rather effective inhibitor for the cytosolic isoform hCA II. The most powerful CA II inhibition effect was found in $\beta$-lactam derivatives of $2 \mathbf{i}$ with a $K_{i}$ value of $0.93 \pm 0.295 \mathrm{nM}$.

(3) The compounds or drugs possessing AChE inhibitory effects are used for the treatment of AD. However, these drugs and compounds have many undesired side effects. Also, the utilization and development of new effective AChEIs is highly desired. Currently the most prescribed AChEIs are Tacrine, Galantamine, Rivastigmine, and Donepezil [55]. In the present study, AChE was very effectively inhibited by $\beta$-lactam derivatives 1-11, with $K_{i}$ value in the range of $0.25 \pm 0.019-1.13 \pm 0.472 \mathrm{nM}$ (Table 2) and calculated from Lineweaver-Burk plots [56]. On the other hand, Tacrine had a $K_{i}$ value of $3.90 \pm 0.792 \mathrm{nM}$.

\section{Materials and Methods}

\subsection{Chemicals}

$\mathrm{CN}-\mathrm{Br}$-activated Sepharose-4B, $p$-nitrophenylacetate, and chemicals for electrophoresis were purchased from Sigma-Aldrich Co. (Steinheim, Germany). All other chemicals were of analytical grade and obtained from Merck (Darmstadt, Germany).

\subsection{General Procedure for the Synthesis of Imines}

2-Amino indane (1 eq) and benzaldehyde (1 eq) were stirred in a beaker for five minutes. The resulting crude imine product was recrystallized from dichloromethane/hexane to give target compound in $95 \%-99 \%$ yield. General synthesis route of novel $\beta$-lactam derivatives (2a-k) is given in Figure 1.

(E)-N-Benzylidene-2,3-dihydro-1H-inden-2-amine (1a). Yield 98\%; ${ }^{1} \mathrm{H}$ NMR $\left(300 \mathrm{MHz} ; \mathrm{ppm} ; \mathrm{CDCl}_{3}\right) \delta$ $3.14(\mathrm{dd}, J=16.05,7.36 \mathrm{~Hz}, 2 \mathrm{H}), 3.21(\mathrm{dd}, J=8.0,16.3 \mathrm{~Hz}, 2 \mathrm{H}), 4.32(\mathrm{p}, J=7.1 \mathrm{~Hz}, 1 \mathrm{H}), 7.16-7.26(\mathrm{~m}$, $4 \mathrm{H}), 7.39-7.43(\mathrm{~m}, 3 \mathrm{H}), 7.74-7.77(\mathrm{~m}, 2 \mathrm{H}), 8.39(1 \mathrm{H}, \mathrm{s}) ;{ }^{13} \mathrm{C} \mathrm{NMR}\left(75 \mathrm{MHz} ; \mathrm{ppm} ; \mathrm{CDCl}_{3}\right) \delta 41.2(2 \mathrm{C})$, $71.5,124.7,126.7$ (2C), 128.4, 128.8, 130.83, 136.5, 142.2 (2C), 160.1 .

(E)-N-(3-Methoxybenzylidene)-2,3-dihydro-1H-inden-2-amine (1b). Yield 96\%; ${ }^{1} \mathrm{H}$ NMR (300 MHz; ppm; $\left.\mathrm{CDCl}_{3}\right) \delta 3.12(\mathrm{dd}, J=15.8,7.0 \mathrm{~Hz}, 2 \mathrm{H}), 3.20(\mathrm{dd}, J=8.41,16.4 \mathrm{~Hz}, 2 \mathrm{H}), 3.82(3 \mathrm{H}, \mathrm{s}), 4.26(\mathrm{p}, J=7.14 \mathrm{~Hz}$, $1 \mathrm{H}), 6.94(\mathrm{dt}, J=7.62,1.66 \mathrm{~Hz}, 1 \mathrm{H}), 7.14-7.35(\mathrm{~m}, 7 \mathrm{H}), 8.33(\mathrm{~s}, 1 \mathrm{H}) ;{ }^{13} \mathrm{C} \mathrm{NMR}\left(75 \mathrm{MHz} ; \mathrm{ppm} ; \mathrm{CDCl}_{3}\right) \delta$ 41.1 (2C), 55.6, 71.4, 111.9, 117.5, 121.7, 124.7 (2C), 126.7 (2C), 129.8 (2C), 137.9, 142.3 (2C), 160.1.

(E)-N-(4-Methylbenzylidene)-2,3-dihydro-1H-inden-2-amine (1c). Yield 97\%; ${ }^{1} \mathrm{H}$ NMR (300 MHz; ppm; $\left.\mathrm{CDCl}_{3}\right) \delta 2.36(3 \mathrm{H}, \mathrm{s}), 3.13(\mathrm{dd}, J=15.3,6.9 \mathrm{~Hz}, 2 \mathrm{H}), 3.17(\mathrm{dd}, J=7.3,15.4 \mathrm{~Hz}, 2 \mathrm{H}), 4.27(\mathrm{p}, J=7.2 \mathrm{~Hz}$, $1 \mathrm{H}), 7.14-7.23(\mathrm{~m}, 6 \mathrm{H}), 7.63(\mathrm{~d}, J=7.99 \mathrm{~Hz}, 2 \mathrm{H}), 8.32(\mathrm{~s}, 1 \mathrm{H}) ;{ }^{13} \mathrm{C} \mathrm{NMR}\left(75 \mathrm{MHz} ; \mathrm{ppm} ; \mathrm{CDCl}_{3}\right) \delta 21.8$, 41.2 (2C), 71.6, 124.7 (2C), 126.7 (2C), 128.4 (2C), 129.5 (2C), 133.8, 141.1, 142.3 (2C), 160.1.

(E)-N-(3-Methylbenzylidene)-2,3-dihydro-1H-inden-2-amine (1d). Yield 97\%; ${ }^{1} \mathrm{H}$ NMR (300 MHz; ppm; $\left.\mathrm{CDCl}_{3}\right) \delta 3.10(\mathrm{dd}, J=16.0,7.4 \mathrm{~Hz}, 2 \mathrm{H}), 3.19(\mathrm{dd}, J=7.6,15.8 \mathrm{~Hz}, 2 \mathrm{H}), 3.81(3 \mathrm{H}, \mathrm{s}), 4.26(\mathrm{p}, J=7.20 \mathrm{~Hz}$, $1 \mathrm{H}), 7.26-7.42(\mathrm{~m}, 6 \mathrm{H}), 7.61-7.63(\mathrm{~d}, J=6.27 \mathrm{~Hz}, 1 \mathrm{H}), 7.73(\mathrm{~s}, 1 \mathrm{H}), 8.43(1 \mathrm{H}, \mathrm{s}) ;{ }^{13} \mathrm{C} \mathrm{NMR}(75 \mathrm{MHz} ; \mathrm{ppm}$; $\mathrm{CDCl}_{3}$ ) $\delta 21.6,41.3(2 \mathrm{C}), 71.7,124.8(2 \mathrm{C}), 126.2,126.8(2 \mathrm{C}), 128.7,128.8,131.8,136.5,138.6,142.3(2 \mathrm{C}), 160.5$.

(E)-N-(3-Chlorobenzylidene)-2,3-dihydro-1H-inden-2-amine (1e). Yield 98\%; ${ }^{1} \mathrm{H}$ NMR (300 MHz; ppm; $\left.\mathrm{CDCl}_{3}\right) \delta 3.19(\mathrm{dd}, J=15.7,6.4 \mathrm{~Hz}, 2 \mathrm{H}), 3.26(\mathrm{dd}, J=7.3,15.7 \mathrm{~Hz}, 2 \mathrm{H}), 4.36(\mathrm{p}, J=6.9 \mathrm{~Hz}, 1 \mathrm{H}), 7.32-7.44$ $(\mathrm{m}, 6 \mathrm{H}), 7.63(\mathrm{~d}, J=6.64 \mathrm{~Hz}, 1 \mathrm{H}), 7.84(1 \mathrm{H}, \mathrm{s}), 8.35(1 \mathrm{H}, \mathrm{s}) ;{ }^{13} \mathrm{C} \mathrm{NMR}\left(75 \mathrm{MHz} ; \mathrm{ppm} ; \mathrm{CDCl}_{3}\right) \delta 41.2(2 \mathrm{C})$, $71.4,124.8$ (2C), 126.8 (2C), 126.8 (2C), 128.0, 130.1, 130.8, 135.0, 138.3, 142.1, 158.6. 
(E)-N-(3,4-Dichlorobenzylidene)-2,3-dihydro-1H-inden-2-amine (1f). Yield 98\%; ${ }^{1} \mathrm{H}$ NMR (300 MHz; ppm; $\left.\mathrm{CDCl}_{3}\right) \delta 3.11(\mathrm{dd}, J=15.6,6.7 \mathrm{~Hz}, 2 \mathrm{H}), 3.18(\mathrm{dd}, J=7.3,15.6 \mathrm{~Hz}, 2 \mathrm{H}), 4.28(\mathrm{p}, J=7.0 \mathrm{~Hz}, 1 \mathrm{H}), 7.15-7.24$ $(\mathrm{m}, 4 \mathrm{H}), 7.35(\mathrm{dd}, J=8.50,2.26 \mathrm{~Hz}, 2 \mathrm{H}), 7.66(\mathrm{dd}, J=8.50,2.26 \mathrm{~Hz}, 2 \mathrm{H}), 8.30(1 \mathrm{H}, \mathrm{s}) ;{ }^{13} \mathrm{C} \mathrm{NMR}(75 \mathrm{MHz}$; ppm; $\mathrm{CDCl}_{3}$ ) $\delta 41.2(2 \mathrm{C}), 71.5,124.8(2 \mathrm{C}), 126.8$ (2C), 129.1 (2C), 129.7 (2C), 134.9, 136.7, $142.1(2 \mathrm{C}), 158.8$.

(E)-N-(3-Bromobenzylidene)-2,3-dihydro-1H-inden-2-amine (1g). Yield 95\%; ${ }^{1} \mathrm{H}$ NMR (300 MHz; ppm; $\left.\mathrm{CDCl}_{3}\right) \delta 3.11(\mathrm{dd}, J=15.6,6.5 \mathrm{~Hz}, 2 \mathrm{H}), 3.19(\mathrm{dd}, J=7.3,15.6 \mathrm{~Hz}, 2 \mathrm{H}), 4.29(\mathrm{p}, J=6.9 \mathrm{~Hz}, 1 \mathrm{H}), 7.15-7.27$ $(\mathrm{m}, 5 \mathrm{H}), 7.51(\mathrm{~d}, J=7.67 \mathrm{~Hz}, 1 \mathrm{H}), 7,61(\mathrm{~d}, J=7.67 \mathrm{~Hz}, 1 \mathrm{H}), 7.93(1 \mathrm{H}, \mathrm{s}), 8.29(1 \mathrm{H}, \mathrm{s}) ;{ }^{13} \mathrm{C} \mathrm{NMR}(75 \mathrm{MHz} ;$ ppm; $\left.\mathrm{CDCl}_{3}\right) \delta 41.1$ (2C), 71.4, 123.1, 124.7 (2C), 126.7 (2C), 127.3, 130.3, 130.9, 133.7, 138.5, 142.1 (2C), 158.5 .

(E)-N-(2-Bromobenzylidene)-2,3-dihydro-1H-inden-2-amine (1h). Yield 97\%; ${ }^{1} \mathrm{H}$ NMR (300 MHz; ppm; $\left.\mathrm{CDCl}_{3}\right) \delta 3.17(\mathrm{dd}, J=15.6,6.4 \mathrm{~Hz}, 2 \mathrm{H}), 3.26(\mathrm{dd}, J=7.2,15.7 \mathrm{~Hz}, 2 \mathrm{H}), 4.42(\mathrm{p}, J=6.8 \mathrm{~Hz}, 1 \mathrm{H}), 7.13-7.36$ $(\mathrm{m}, 6 \mathrm{H}), 7.59(\mathrm{dd}, J=7.67,1.69 \mathrm{~Hz}, 1 \mathrm{H}), 8.08(\mathrm{dd}, J=7.66,1.43 \mathrm{~Hz}, 1 \mathrm{H}), 8.77(1 \mathrm{H}, \mathrm{s}) .{ }^{13} \mathrm{C} \mathrm{NMR}(75 \mathrm{MHz} ;$ ppm; $\left.\mathrm{CDCl}_{3}\right) \delta 41.2$ (2C), 71.3, 124.7 (2C), 125.2, 126.7 (2C), 127.8, 129.2, 131.9, 133.2, 134.8, 142.1 (2C), 159.1 .

(E)-N-(4-Bromobenzylidene)-2,3-dihydro-1H-inden-2-amine (1i). Yield 98\%; ${ }^{1} \mathrm{H}$ NMR (300 MHz; ppm; $\left.\mathrm{CDCl}_{3}\right) \delta 3.11(\mathrm{dd}, J=15.6,6.6 \mathrm{~Hz}, 2 \mathrm{H}), 3.19(\mathrm{dd}, J=7.3,15.6 \mathrm{~Hz}, 2 \mathrm{H}), 4.29(\mathrm{p}, J=6.9 \mathrm{~Hz}, 1 \mathrm{H}), 7.15-7.24$ $(\mathrm{m}, 4 \mathrm{H}), 7.52(\mathrm{dd}, J=8.4,5.0 \mathrm{~Hz}, 2 \mathrm{H}), 7.59(\mathrm{dd}, J=8.6,4.8 \mathrm{~Hz}, 2 \mathrm{H}), 8.30(1 \mathrm{H}, \mathrm{s}) .{ }^{13} \mathrm{C}$ NMR $(75 \mathrm{MHz} ; \mathrm{ppm}$; $\mathrm{CDCl}_{3}$ ) $\delta 41.1(2 \mathrm{C}), 71.4,124.7(2 \mathrm{C}), 125.1,126.7(2 \mathrm{C}), 129.8(2 \mathrm{C}), 132.0(2 \mathrm{C}), 135.3,142.1(2 \mathrm{C}), 158.9$.

(E)-N-(2-Nitrobenzylidene)-2,3-dihydro-1H-inden-2-amine (1j). Yield 97\%; ${ }^{1} \mathrm{H}$ NMR (300 MHz; ppm; $\left.\mathrm{CDCl}_{3}\right) \delta 3.13(\mathrm{dd}, J=15.7,5.9 \mathrm{~Hz}, 2 \mathrm{H}), 3.25(\mathrm{dd}, J=7.2,15.7 \mathrm{~Hz}, 2 \mathrm{H}), 4.39(\mathrm{p}, J=6.6 \mathrm{~Hz}, 1 \mathrm{H})$, $7.15-7.25(\mathrm{~m}, 4 \mathrm{H}), 7.56(\mathrm{dt}, J=7.46,1.54 \mathrm{~Hz}, 2 \mathrm{H}), 8.02(\mathrm{dd}, J=7.71,1.39 \mathrm{~Hz}, 2 \mathrm{H}), 8.9(1 \mathrm{H}, \mathrm{s}) ;{ }^{13} \mathrm{C}$ NMR (75 MHz; ppm; $\mathrm{CDCl}_{3}$ ) $\delta 41.1$ (2C), 71.2, 124.5, 124.8 (2C), 126.8 (2C), 130.1, 130.8, 131.5, 133.7, 141.9 (2C), 149.0, 156.2.

(E)-N-(4-Fluorobenzylidene)-2,3-dihydro-1H-inden-2-amine (1k). Yield 94\%; ${ }^{1} \mathrm{H}$ NMR (300 MHz; ppm; $\left.\mathrm{CDCl}_{3}\right) \delta 3.11(\mathrm{dd}, J=15.6,6.7 \mathrm{~Hz}, 2 \mathrm{H}), 3.18(\mathrm{dd}, J=7.3,15.6 \mathrm{~Hz}, 2 \mathrm{H}), 4.28(\mathrm{p}, J=7.0 \mathrm{~Hz}, 1 \mathrm{H}), 7.03-7.24$ $(\mathrm{m}, 6 \mathrm{H}), 7.71(\mathrm{dd}, J=4.9,5.5 \mathrm{~Hz}, 2 \mathrm{H}), 7.59(\mathrm{dd}, J=8.6,4.9 \mathrm{~Hz}, 2 \mathrm{H}), 8.32(1 \mathrm{H}, \mathrm{s}) ;{ }^{13} \mathrm{C} \mathrm{NMR}(75 \mathrm{MHz} ; \mathrm{ppm}$; $\left.\mathrm{CDCl}_{3}\right) \delta 41.2(2 \mathrm{C}), 71.3,115.7(2 \mathrm{C}), 116.0(2 \mathrm{C}), 124.7(2 \mathrm{C}), 126.7$ (2C), 130.2, 130.3, $142.2(2 \mathrm{C}), 158.6$.

\subsection{General Procedure for the Synthesis of $\beta$-Lactams}

To a solution of imine (1 eq) and triethylamine ( $3 \mathrm{eq})$ in dichloromethane, a solution of acetoxyacetyl chloride (2 eq) in dichloromethane was added dropwise over a period of $10 \mathrm{~min}$ at room temperature. The reaction mixture was then stirred for an additional $1 \mathrm{~h}$ at room temperature. The mixture was concentrated, then extracted with Ethyl Acetate and dried over Magnesium Sulphate; the solvent was removed in a vacuum. Obtained product was purified over a silica gel packed column chromatography using Hexane: EtOAc $(1: 1 v / v)$. The purified product was dried under vacuo and recrystallized from Ethanol yields $\beta$-lactam derivatives ( $70 \%-93 \%$ yield).

\subsection{Spectral Data}

$\left(3 S^{*}, 4 R^{*}\right)-1-\left(2,3-D i h y d r o-1 H\right.$-inden-2-yl)-2-oxo-4-phenylazetidin-3-yl acetate (2a). Yield 91\%, m.p. $171-173{ }^{\circ} \mathrm{C}$; ${ }^{1} \mathrm{H}$ NMR $\left(300 \mathrm{MHz} ; \mathrm{ppm} ; \mathrm{CDCl}_{3}\right) \delta 1.65(3 \mathrm{H}, \mathrm{s}), 2.88(\mathrm{dd}, J=15.8,6.1 \mathrm{~Hz}, 1 \mathrm{H}), 2.89(\mathrm{dd}, J=15.8,7.1 \mathrm{~Hz}$, $1 \mathrm{H}), 3.14-3.28(\mathrm{~m}, 2 \mathrm{H}), 4.54(\mathrm{p}, J=7.0 \mathrm{~Hz}, 1 \mathrm{H}), 4.81(\mathrm{~d}, J=4.6 \mathrm{~Hz}, 1 \mathrm{H}), 5.72(\mathrm{~d}, J=4.6 \mathrm{~Hz}, 1 \mathrm{H}), 6.93-6.96$ (m, 1H), 7.04-7.21 (m, 5H), 7.29-7.31 (m, 3H); $\left.{ }^{13} \mathrm{C} \mathrm{NMR} \mathrm{(75} \mathrm{MHz;} \mathrm{ppm;} \mathrm{CDCl}_{3}\right) \delta$ 20.1, 37.3, 37.4, 54.0, 61.7, 76.6, 124.6 (2C), 127.0 (2C), 128.3 (2C), 128.5 (2C), 128.9, 133.7, 140.3, 140.3, 165.3, 169.2; MS: $\mathrm{m} / \mathrm{z}$ $344.10[\mathrm{M}+\mathrm{Na}]^{+}$.

$\left(3 S^{*}, 4 R^{*}\right)-1-(2,3-D i h y d r o-1 H$-inden-2-yl)-2-(3-methoxyphenyl)-4-oxoazetidin-3-yl acetate (2b). Yield 70\%, m.p. 98-100 ${ }^{\circ} \mathrm{C} ;{ }^{1} \mathrm{H} \mathrm{NMR}\left(300 \mathrm{MHz} ; \mathrm{ppm} ; \mathrm{CDCl}_{3}\right) \delta 1.70(3 \mathrm{H}, \mathrm{s}), 2.90(\mathrm{dd}, J=15.9,6.1 \mathrm{~Hz}, 1 \mathrm{H}), 2.99$ $(\mathrm{dd}, J=15.8,7.1 \mathrm{~Hz}, 1 \mathrm{H}), 3.13-3.27(\mathrm{~m}, 2 \mathrm{H}), 3.75(\mathrm{~s}, 3 \mathrm{H}), 4.54(\mathrm{p}, J=6.6 \mathrm{~Hz}, 1 \mathrm{H}), 4.77(\mathrm{~d}, J=4.7 \mathrm{~Hz}, 1 \mathrm{H})$, 
$5.72(\mathrm{~d}, J=4.7 \mathrm{~Hz}, 1 \mathrm{H}), 6.66-6.67(1 \mathrm{H}, \mathrm{m}), 6.77-6.85(2 \mathrm{H}, \mathrm{m}), 6.96(\mathrm{~d}, J=6.94 \mathrm{~Hz}, 1 \mathrm{H}), 7.04-727(\mathrm{~m}, 4 \mathrm{H})$; ${ }^{13} \mathrm{C}$ NMR $\left(75 \mathrm{MHz} ; \mathrm{ppm} ; \mathrm{CDCl}_{3}\right.$ ) $\delta$ 20.2, 37.3, 37.4, 54.0, 55.4, 61.6, 76.5, 113.8, 114.6, 120.8, 124.5, 124.6 (2C), 127.0 (2C), 129.4, 135.4, 140.4, 159.5, 165.2, 169.3; MS: $m / z 344.16[\mathrm{M}+\mathrm{Na}]^{+}$.

(3S* $\left.4 R^{*}\right)-1$-(2,3-Dihydro-1H-inden-2-yl)-2-oxo-4-p-tolylazetidin-3-yl acetate (2c). Yield 92\%, m.p. 113-115 ${ }^{\circ} \mathrm{C}$; ${ }^{1} \mathrm{H}$ NMR (300 MHz; ppm; $\left.\mathrm{CDCl}_{3}\right) \delta 1.68(3 \mathrm{H}, \mathrm{s}), 2.29(3 \mathrm{H}, \mathrm{s}), 2.88(\mathrm{dd}, J=15.8,6.0 \mathrm{~Hz}, 1 \mathrm{H}), 2.98(\mathrm{dd}$, $J=15.8,7.1 \mathrm{~Hz}, 1 \mathrm{H}), 3.13-3.27(\mathrm{~m}, 2 \mathrm{H}), 4.53(\mathrm{p}, J=6.6 \mathrm{~Hz}, 1 \mathrm{H}), 4.76(\mathrm{~d}, J=4.6 \mathrm{~Hz}, 1 \mathrm{H}), 5.71(\mathrm{~d}, J=4.6 \mathrm{~Hz}$, 1H), 6.92-7.27 (m, 8H); $\left.{ }^{13} \mathrm{C} \mathrm{NMR} \mathrm{(75} \mathrm{MHz;} \mathrm{ppm;} \mathrm{CDCl}_{3}\right) \delta 20.1,21.5,37.3,37.4,54.0,61.7,76.5,124.5$, 125.6, 127.0 (2C), 128.1, 129.3, 129.6, 133.6, 138.0, 140.4 (2C), 165.3, 169.3; MS: $m / z 358.04[\mathrm{M}+\mathrm{Na}]^{+}$.

(3S* $\left.4 R^{*}\right)$-1-(2,3-Dihydro-1H-inden-2-yl)-2-oxo-4-m-tolylazetidin-3-yl acetate (2d). Yield 87\%, m.p. 96-98 ${ }^{\circ} \mathrm{C}$; ${ }^{1} \mathrm{H}$ NMR (300 MHz; ppm; $\left.\mathrm{CDCl}_{3}\right) \delta 1.69(3 \mathrm{H}, \mathrm{s}), 2.34(3 \mathrm{H}, \mathrm{s}), 2.87(\mathrm{dd}, J=15.8,6.4 \mathrm{~Hz}, 1 \mathrm{H}), 2.97(\mathrm{dd}$, $J=15.7,7.3 \mathrm{~Hz}, 1 \mathrm{H}), 3.18(\mathrm{~m}, J=7.2,15.3 \mathrm{~Hz}, 1 \mathrm{H}), 3.22(\mathrm{~m}, J=6.3,15.3 \mathrm{~Hz}, 1 \mathrm{H}), 3.75(3 \mathrm{H}, \mathrm{s}), 4.51(\mathrm{p}$, $J=6.8 \mathrm{~Hz}, 1 \mathrm{H}), 4.79(\mathrm{~d}, J=4.6 \mathrm{~Hz}, 1 \mathrm{H}), 6.95-6.97(\mathrm{~m}, 1 \mathrm{H}), 7.02-7.17(\mathrm{~m}, 7 \mathrm{H}) ;{ }^{13} \mathrm{C}$ NMR $(75 \mathrm{MHz} ; \mathrm{ppm}$; $\left.\mathrm{CDCl}_{3}\right) \delta 20.2,21.4,37.2,37.3,53.9,61.6,76.6,124.6(2 \mathrm{C}), 127.0,127.0,128.5(2 \mathrm{C}), 129.0(2 \mathrm{C}), 130.6,138.7$, 140.4, 140.4, 165.4, 169.4; MS: $m / z 358.09[\mathrm{M}+\mathrm{Na}]^{+}$.

(3S*,4R*)-2-(3-Chlorophenyl)-1-(2,3-dihydro-1H-inden-2-yl)-4-oxoazetidin-3-yl acetate (2e). Yield 98\%, m.p. $115-117^{\circ} \mathrm{C} ;{ }^{1} \mathrm{H}$ NMR $\left(300 \mathrm{MHz} ; \mathrm{ppm} ; \mathrm{CDCl}_{3}\right) \delta 1.78(3 \mathrm{H}, \mathrm{s}), 2.83(\mathrm{dd}, J=15.9,7.0 \mathrm{~Hz}, 1 \mathrm{H}), 3.00(\mathrm{dd}$, $J=15.9,5.2 \mathrm{~Hz}, 1 \mathrm{H}), 3.10-3.24(\mathrm{~m}, 2 \mathrm{H}), 4.57(\mathrm{p}, J=6.1 \mathrm{~Hz}, 1 \mathrm{H}), 4.72(\mathrm{~d}, J=4.6 \mathrm{~Hz}, 1 \mathrm{H}), 5.71(\mathrm{~d}, J=4.6 \mathrm{~Hz}$, 1H), 6.89-6.91 (m, 1H), 7.01-7.28 (m, 7H); ${ }^{13} \mathrm{C}$ NMR (75 MHz; ppm; $\left.\mathrm{CDCl}_{3}\right) \delta$ 20.1, 37.5, 37.6, 54.0, 61.1, 76.6, 124.5, 124.6, 126.7, 127.1, 127.2, 128.6, 129.1, 129.5, 134.3, 136.0, 140.2, 164.9, 169.2; MS: $\mathrm{m} / \mathrm{z} 358.20$ $[\mathrm{M}+2 \mathrm{H}]^{+}$.

$\left(3 S^{*}, 4 R^{*}\right)-2-(3,4-D i c h l o r o p h e n y l)-1-(2,3-d i h y d r o-1 H-i n d e n-2-y l)-4-o x o a z e t i d i n-3-y l$ acetate (2f). Yield 84\%, m.p. ${ }^{138-140}{ }^{\circ} \mathrm{C} ;{ }^{1} \mathrm{H}$ NMR $\left(300 \mathrm{MHz} ; \mathrm{ppm} ; \mathrm{CDCl}_{3}\right) \delta 1.77(3 \mathrm{H}, \mathrm{s}), 2.79(\mathrm{dd}, J=4.4,16.0 \mathrm{~Hz}, 1 \mathrm{H})$, $3.01(\mathrm{dd}, J=16.0,3.8 \mathrm{~Hz}, 1 \mathrm{H}), 3.10(\mathrm{dd}, J=6.6,17.1 \mathrm{~Hz}, 1 \mathrm{H}), 3.18(\mathrm{dd}, J=4.6,16.0 \mathrm{~Hz}, 1 \mathrm{H}), 4.56(\mathrm{p}$, $J=6.2 \mathrm{~Hz}, 1 \mathrm{H}), 4.76(\mathrm{~d}, J=4.6 \mathrm{~Hz}, 1 \mathrm{H}), 5.70(\mathrm{~d}, J=4.6 \mathrm{~Hz}, 1 \mathrm{H}), 6.87-6.90(\mathrm{~m}, 1 \mathrm{H}), 6.97(\mathrm{~d}, J=8.28 \mathrm{~Hz}$, 1H), 7.04-7.14 (m, 4H), $7.33(\mathrm{~d}, J=8.28 \mathrm{~Hz}, 1 \mathrm{H}) ;{ }^{13} \mathrm{C} \mathrm{NMR}\left(75 \mathrm{MHz} ; \mathrm{ppm} ; \mathrm{CDCl}_{3}\right) \delta 20.3,37.5,37.9$, 54.0, 60.5, 76.5, 124.5, 124.6, 127.2, 127.3, 127.7, 130.2, 130.5, 132.6, 133.0, 134.3, 140.1, 140.1, 164.8, 169.2; MS: $m / z 392.20[\mathrm{M}+\mathrm{H}]^{+}$.

$\left(3 S^{*}, 4 R^{*}\right)$-2-(3-Bromophenyl)-1-(2,3-dihydro-1H-inden-2-yl)-4-oxoazetidin-3-yl acetate (2g). Yield 78\%, m.p. 132-134 ${ }^{\circ} \mathrm{C} ;{ }^{1} \mathrm{H}$ NMR $\left(300 \mathrm{MHz} ; \mathrm{ppm} ; \mathrm{CDCl}_{3}\right) \delta 1.73(3 \mathrm{H}, \mathrm{s}), 2.83(\mathrm{dd}, J=4.9,16.0 \mathrm{~Hz}, 1 \mathrm{H}), 3.00(\mathrm{dd}$, $J=15.9,7.0 \mathrm{~Hz}, 1 \mathrm{H}), 3.17(\mathrm{ddd}, J=5.2,6.5,15.6 \mathrm{~Hz}, 2 \mathrm{H}), 4.58(\mathrm{p}, J=6.0 \mathrm{~Hz}, 1 \mathrm{H}), 4.70(\mathrm{~d}, J=4.6 \mathrm{~Hz}$, $1 \mathrm{H}), 5.70(\mathrm{~d}, J=4.6 \mathrm{~Hz}, 1 \mathrm{H}), 6.89-6.92(\mathrm{~m}, 1 \mathrm{H}), 7.05-7.17(\mathrm{~m}, 4 \mathrm{H}), 7.26-7.27(\mathrm{~m}, 2 \mathrm{H}), 7.40-7.43(\mathrm{~m}, 1 \mathrm{H})$; ${ }^{13} \mathrm{C} \mathrm{NMR}\left(75 \mathrm{MHz} ; \mathrm{ppm} ; \mathrm{CDCl}_{3}\right) \delta$ 20.1, 37.5, 37.6, 54.0, 61.0, 76.5, 122.3, 124.5, 124.5, 127.1, 127.3, 129.8, $131.5,132.0,136.3,140.2,140.2,164.9,169.2 ; \mathrm{MS}: m / z 424.05[\mathrm{M}+\mathrm{H}+\mathrm{Na}]^{+}$.

$\left(3 S^{*}, 4 R^{*}\right)$-2-(2-Bromophenyl)-1-(2,3-dihydro-1H-inden-2-yl)-4-oxoazetidin-3-yl acetate (2h). Yield 83\%, m.p. 123-125 ${ }^{\circ} \mathrm{C} ;{ }^{1} \mathrm{H}$ NMR $\left(300 \mathrm{MHz} ; \mathrm{ppm} ; \mathrm{CDCl}_{3}\right) \delta 1.69(3 \mathrm{H}, \mathrm{s}), 2.92(\mathrm{dd}, J=6.0,15.8 \mathrm{~Hz}, 1 \mathrm{H}), 3.02(\mathrm{dd}$, $J=15.8,7.0 \mathrm{~Hz}, 1 \mathrm{H}), 3.22(\mathrm{~d}, J=6.2 \mathrm{~Hz}, 2 \mathrm{H}), 4.53(\mathrm{p}, J=6.5 \mathrm{~Hz}, 1 \mathrm{H}), 5.40(\mathrm{~d}, J=4.7 \mathrm{~Hz}, 1 \mathrm{H}), 5.85(\mathrm{~d}$, $J=4.7 \mathrm{~Hz}, 1 \mathrm{H}), 6.99-7.01(\mathrm{~m}, 1 \mathrm{H}), 7.06-7.19(\mathrm{~m}, 4 \mathrm{H}), 7.29-7.38(\mathrm{~m}, 2 \mathrm{H}), 7.47-7.50(\mathrm{~m}, 1 \mathrm{H}) ;{ }^{13} \mathrm{C} \mathrm{NMR}$ (75 MHz; ppm; $\mathrm{CDCl}_{3}$ ) $\delta 20.1,37.2,37.5,54.2,60.6,76.0,124.5,124.6,124.7,126.9,127.1,127.2,129.4$, 130.1, 133.0, 133.3, 140.1, 140.2, 165.5, 168.9; MS: $m / z$ $422.03[\mathrm{M}+\mathrm{Na}]^{+}$.

(3S*,4R*)-2-(4-Bromophenyl)-1-(2,3-dihydro-1H-inden-2-yl)-4-oxoazetidin-3-yl acetate (2i). Yield 76\%, m.p. 114-116 ${ }^{\circ} \mathrm{C} ;{ }^{1} \mathrm{H}$ NMR (300 MHz; ppm; $\left.\mathrm{CDCl}_{3}\right) \delta 1.73(3 \mathrm{H}, \mathrm{s}), 2.83(\mathrm{dd}, J=5.3,15.9 \mathrm{~Hz}, 1 \mathrm{H}), 2.99(\mathrm{dd}$, $J=15.9,7.0 \mathrm{~Hz}, 1 \mathrm{H}), 3.15(\mathrm{~m}, J=5.7,13.2 \mathrm{~Hz}, 1 \mathrm{H}), 3.18(\mathrm{~m}, J=6.6,13.2 \mathrm{~Hz}, 1 \mathrm{H}), 4.56(\mathrm{p}, J=6.2 \mathrm{~Hz}, 1 \mathrm{H})$, $4.74(\mathrm{~d}, J=4.6 \mathrm{~Hz}, 1 \mathrm{H}), 6.92(\mathrm{~m}, 1 \mathrm{H}), 7.02(\mathrm{~d}, J=8.28 \mathrm{~Hz}, 2 \mathrm{H}), 7.06-7.16(3 \mathrm{H}, \mathrm{m}), 7.41(\mathrm{~d}, J=8.41 \mathrm{~Hz}$, 2H); ${ }^{13} \mathrm{C}$ NMR $\left(75 \mathrm{MHz} ; \mathrm{ppm} ; \mathrm{CDCl}_{3}\right) \delta 20.2,37.5,37.5,54.0,61.1,76.5,122.9,124.5,124.6,127.1(2 \mathrm{C})$, 130.1 (2C), $131.5(2 \mathrm{C}), 133.0,140.2,140.2,165.0,169.2 ; \mathrm{MS}: \mathrm{m} / z 421.92[\mathrm{M}+\mathrm{Na}]^{+}$.

$\left(3 S^{*}, 4 R^{*}\right)-1-(2,3-D i h y d r o-1 H$-inden-2-yl)-2-(2-nitrophenyl)-4-oxoazetidin-3-yl acetate (2j). Yield 93\%, m.p. $117-119{ }^{\circ} \mathrm{C} ;{ }^{1} \mathrm{H}$ NMR $\left(300 \mathrm{MHz} ; \mathrm{ppm} ; \mathrm{CDCl}_{3}\right) \delta 1.70(3 \mathrm{H}, \mathrm{s}), 2.99(\mathrm{dd}, J=5.6,15.9 \mathrm{~Hz}, 1 \mathrm{H}), 3.08(\mathrm{dd}$, 
$J=15.8,6.9 \mathrm{~Hz}, 1 \mathrm{H}), 3.25(\mathrm{~d}, J=6.1 \mathrm{~Hz}, 2 \mathrm{H}), 4.57(\mathrm{p}, J=6.6 \mathrm{~Hz}, 1 \mathrm{H}), 5.59(\mathrm{~d}, J=5.2 \mathrm{~Hz}, 1 \mathrm{H}), 6.04(\mathrm{~d}$, $J=5.2 \mathrm{~Hz}, 1 \mathrm{H}), 6.97-6.99(\mathrm{~m}, 1 \mathrm{H}), 7.04-7.12(\mathrm{~m}, 3 \mathrm{H}), 7.46-7.52(\mathrm{~m}, 1 \mathrm{H}), 7.59-7.63(\mathrm{~m}, 2 \mathrm{H}), 7.95-7.98(\mathrm{~m}$, $1 \mathrm{H}) ;{ }^{13} \mathrm{C}$ NMR (75 MHz; ppm; $\mathrm{CDCl}_{3}$ ) $\delta 20.1,37.2,37.7,54.7,57.4,76.4,124.4,124.6,125.3,127.2,127.4$, $129.4,129.5,130.3,132.9,139.9,140.1,149.0,165.9,168.6 ; \mathrm{MS}: \mathrm{m} / z$ 388,93 [M + Na] ${ }^{+}$.

$\left(3 S^{*}, 4 R^{*}\right)-1-(2,3-D i h y d r o-1 H-i n d e n-2-y l)-2-(4-f l u o r o p h e n y l)-4-o x o a z e t i d i n-3-y l$ acetate (2k). Yield 91\%, m.p. 114-116 ${ }^{\circ} \mathrm{C} ;{ }^{1} \mathrm{H}$ NMR $\left(300 \mathrm{MHz} ; \mathrm{ppm} ; \mathrm{CDCl}_{3}\right) \delta 1.71(3 \mathrm{H}, \mathrm{s}), 2.84(\mathrm{dd}, J=5.5,15.8 \mathrm{~Hz}, 1 \mathrm{H}), 2.99$ (dd, $J=15.9,7.1 \mathrm{~Hz}, 1 \mathrm{H}), 3.18(\mathrm{~d}, J=6.2 \mathrm{~Hz}, 2 \mathrm{H}), 4.56(\mathrm{p}, J=6.3 \mathrm{~Hz}, 1 \mathrm{H}), 4.77(\mathrm{~d}, J=4.6 \mathrm{~Hz}, 1 \mathrm{H}), 5.70(\mathrm{~d}$, $J=4.6 \mathrm{~Hz}, 1 \mathrm{H}), 6.91-6.99(\mathrm{~m}, 3 \mathrm{H}), 7.04-7.16(\mathrm{~m}, 5 \mathrm{H}) ;{ }^{13} \mathrm{C} \mathrm{NMR}\left(75 \mathrm{MHz} ; \mathrm{ppm} ; \mathrm{CDCl}_{3}\right) \delta 20.1,37.4,37.5$, 53.9, 61.0, 76.6, 115.2, 115.5, 124.5, 124.5, 127.1 (2C), 129.6, 129.6, 130.2, 130.3, 140.2 (2C), 165.1, 169.2; MS: $m / z 344.13[\mathrm{M}+\mathrm{Na}]^{+}$.

\subsection{Biochemical Assays}

Erythrocytes were obtained from the Research Hospital at Atatürk University. The red cells were haemolysed with ice-cold water after washing with $0.9 \% \mathrm{NaCl}$. The hemolysate was applied to the prepared Sepharose-4B-tyrosine-sulfanylamide affinity gel [57]. Both CA isoenzymes were purified by Sepharose-4B-L-tyrosine-sulfanilamide affinity chromatography in a single step [58-60].Sepharose-4B-L-tyrosine-sulfanilamide was prepared according to a previous method. Thus, homogenate solution acidity was adjusted and supernatant was transferred to the previously prepared Sepharose-4B-L-tyrosine-sulphanilamide affinity column [61,62]. The proteins flow in the column eluates was spectrophotometrically determined at $280 \mathrm{~nm}$. All purification steps were performed at $4{ }^{\circ} \mathrm{C}$. Protein quantity was determined at $595 \mathrm{~nm}$ according to the Bradford method [63]. Bovine serum albumin was used as the standard protein [64-66]. To monitor purification of both isoenzymes, sodium dodecyl sulphate-polyacrylamide gel electrophoresis (SDS-PAGE) was used according to the procedure of Laemmli [67]. In this application, the imaging method was performed in $10 \%$ and $3 \%$ acrylamide for the running and the stacking gel, respectively, with $0.1 \%$ SDS [68-70].

Enzyme activity was determined hydrolysis of $p$-nitrophenylacetate (PNA) to $p$-nitrophenolate at $348 \mathrm{~nm}$ according to the method of Verpoorte et al. [71] and described previously. The inhibitory effects of $\beta$-lactam derivatives $\mathbf{2 a}-\mathbf{k}$ were examined. To obtain the half maximal inhibitory concentration $\left(\mathrm{IC}_{50}\right)$ values, CA I, and II activity was measured in the presence of $\beta$-lactam derivatives at different cuvette concentrations. Activity (\%)-[ $\beta$-lactam] graph was drawn for each of the $\beta$-lactam derivatives 2a-k [72-74]. To determine Ki values, three different $\beta$-lactam derivative concentrations were tested. In these experiments, different substrate (PNA) concentrations were used and Lineweaver-Burk curves were drawn [56] as previously described [54].

The inhibition effects of $\beta$-lactam derivatives $\mathbf{2 a}-\mathbf{k}$ on $\mathrm{AChE}$ activities were measured according to the Ellman's method [75] described previously [76]. Acetylthiocholine iodide (AChI) and 5,5'-dithio-bis(2-nitro-benzoic) acid (DTNB) were used as substrate. To this end, $100 \mu \mathrm{L}$ of Tris/ $\mathrm{HCl}$ buffer $(1 \mathrm{M}, \mathrm{pH} 8.0)$ and $10 \mu \mathrm{L}$ of $\beta$-lactam derivative solution at different concentrations and $50 \mu \mathrm{L}$ $\operatorname{AChE}\left(5.32 \times 10^{-3} \mathrm{U}\right)$ solution were mixed and incubated for $10 \mathrm{~min}$ at $25{ }^{\circ} \mathrm{C}$. Then $50 \mu \mathrm{L}$ of DTNB $(0.5 \mathrm{mM})$ was transferred. Then the reaction was initiated by the addition of $50 \mu \mathrm{L}$ of AChI. The hydrolysis of $\mathrm{AChI}$ was recorded spectrophotometrically by the formation of 5-thio-2-nitrobenzoate anion as the result of the reaction of DTNB with thiocholine at a wavelength of $412 \mathrm{~nm}$ [77]. The $\mathrm{IC}_{50}$ values were determined by spectrophotometric measurement of the effect of increasing test compound ( $\beta$-lactam derivatives $\mathbf{2 a}-\mathbf{k}$ ) concentrations on $\mathrm{AChE}$ activity. The $\mathrm{IC}_{50}$ and $K_{i}$ values are calculated in the same way as for CA isoenzymes. Tacrine was used as positive control.

\section{Conclusions}

The hCA I, and II isoenzymes were inhibited by $\beta$-lactams $\mathbf{2 a}-\mathbf{k}$ at different functional groups $\left(\mathrm{CH}_{3}, \mathrm{NO}_{2}, \mathrm{Br}, \mathrm{F}, \mathrm{Cl}\right.$, and phenol) in the micromolar range. These compounds have shown nanomolar inhibition against both cytosolic hCA I, and II. These results indicate that the $\beta$-lactam ring and 
derivatives may be new CA inhibitors in addition to the well-known sulphonamides. Also, AChE was potently inhibited by $\beta$-lactams $2 \mathbf{a}-\mathbf{k}$ with $K_{i}$ values in the range of $0.25 \pm 0.019-1.13 \pm 0.472 \mathrm{nM}$.

Supplementary Materials: Supplementary materials can be found at www.mdpi.com/1422-0067/17/10/1736/s1.

Acknowledgments: We gratefully acknowledge the partial financial support for this research project from Sakarya University BAPK-2012-02-04-031.

Author Contributions: Mustafa Zengin and İlhami Gülçin were responsible for the majority of this work, including the execution of experiments, data analysis, and writing and publication of this report; Hayriye Genç synthesized the 2-aminoindan $\beta$-lactam derivatives; Zeynep Köksal, Ramazan Kalin and Nastaran Sadeghian performed carbonic anhydrase isoenzymes inhibition assays; Umit M. Kocyigit and Hasan Özdemir analyzed acetylcholinesterase inhibitors data. All authors have read and approved the final manuscript.

Conflicts of Interest: The authors declare no conflict of interest.

\section{References}

1. Huang, Z.; Pan, X.D.; Huang, B.; Xu, J.J.; Wang, M.L.; Ren, Y.P. Determination of $15 \beta$-lactam antibiotics in pork muscle by matrix solid-phase dispersion extraction (MSPD) and ultra-high pressure liquid chromatography tandem mass spectrometry. Food Control 2016, 66, 145-150. [CrossRef]

2. Zhang, L.; Jin, Y.; Mao, H.; Zheng, L.; Zhao, J.; Peng, Y.; Du, S.; Zhang, Z. Structure-selective hot-spot Raman enhancement for direct identification and detection of trace penicilloic acid allergen in penicillin. Biosens. Bioelectron. 2014, 58, 165-171. [CrossRef] [PubMed]

3. Vijay Kumar, M.M.J.; Yogananda, R.; Snehalatha Shameer, H.; Jayachandran, E.; Sreenivasa, G.M. Synthesis and characterization of novel $\mathrm{N}$-substituted-3-chloro-2-azetidinones as potential anticonvulsant agents. J. Biomed. Sci. Res. 2009, 1, 1-10.

4. Banik, I.; Becker, F.F.; Banik, B.K. Stereoselective synthesis of $\beta$-lactams with polyaromatic imines: Entry to new and novel anticancer agents. J. Med. Chem. 2003, 46, 12-15. [CrossRef] [PubMed]

5. Clemente, A.; Domingos, A.; Grancho, A.P.; Iley, J.; Moreira, R.; Neres, J.; Palma, N.; Santana, A.B.; Valente, E. Design, synthesis and stability of $\mathrm{N}$-acyloxymethyl- and $\mathrm{N}$-aminocarbonyloxymethyl-2-azetidinones as human leukocyte elastase inhibitors. Bioorg. Med. Chem. Lett. 2001, 11, 1065-1068. [CrossRef]

6. Bytyqi-Damoni, A.; Genç, H.; Zengin, M.; Beyaztas, S.; Gençer, N.; Arslan, O. In vitro effect of novel $\beta$-lactam compounds on xanthine oxidase enzyme activity. Artif. Cells Blood Substit. Immobil. Biotechnol. 2012, 40, 369-377. [CrossRef] [PubMed]

7. Küçük, M.; Gulcin, I. Purification and characterization of carbonic anhydrase enzyme from black sea trout (Salmo trutta Labrax Coruhensis) kidney and inhibition effects of some metal ions on the enzyme activity. Environ. Toxicol. Pharmacol. 2016, 44, 134-139. [CrossRef] [PubMed]

8. Troisi, L.; Granito, C.; Pindinelli, E. Novel and recent synthesis and applications of $\beta$-lactams. Top. Heterocycl. Chem. 2010, 22, 101-209.

9. Taslimi, P.; Gulcin, I.; Ozgeris, B.; Goksu, S.; Tumer, F.; Alwasel, S.H.; Supuran, C.T. The human carbonic anhydrase isoenzymes I and II (hCA I and II) inhibition effects of trimethoxyindane derivatives. J. Enzym. Inhib. Med. Chem. 2016, 31, 152-157. [CrossRef] [PubMed]

10. Scozzafava, A.; Kalın, P.; Supuran, C.T.; Gülçin, I.; Alwasel, S. The impact of hydroquinone on acetylcholine esterase and certain human carbonic anhydrase isoenzymes (hCA I, II, IX, and XII). J. Enzym. Inhib. Med. Chem. 2015, 30, 941-946. [CrossRef] [PubMed]

11. Artunç, T.; Çetinkaya, Y.; Göçer, H.; Gulcin, I.; Menzek, A.; Sahin, E.; Supuran, C.T. Synthesis of 4-[2-(3,4-dimethoxybenzyl)cyclopentyl]-1,2-dimethoxybenzene derivatives and evaluations of their carbonic anhydrase isoenzymes inhibitory effects. Chem. Biol. Drug Des. 2016, 87, 594-607. [CrossRef] [PubMed]

12. Akıncıoğlu, A.; Akıncıoğlu, H.; Gülçin, I.; Durdağı, S.; Supuran, C.T.; Göksu, S. Discovery of potent carbonic anhydrase and acetylcholine esterase inhibitors: Novel sulfamoylcarbamates and sulfamides derived from acetophenones. Bioorg. Med. Chem. 2015, 23, 3592-3602. [CrossRef] [PubMed]

13. Yıldırım, A.; Atmaca, U.; Keskin, A.; Topal, M.; Çelik, M.; Gülçin, I.; Supuran, C.T. N-Acylsulfonamides strongly inhibit human carbonic anhydrase isoenzymes I and II. Bioorg. Med. Chem. 2015, 23, 2598-2605. [CrossRef] [PubMed] 
14. Boztaş, M.; Çetinkaya, Y.; Topal, M.; Gülçin, İ.; Menzek, A.; Şahin, E.; Tanc, M.; Supuran, C.T. Synthesis and carbonic anhydrase isoenzymes I, II, IX, and XII inhibitory effects of dimethoxy-bromophenol derivatives incorporating cyclopropane moieties. J. Med. Chem. 2015, 58, 640-650. [CrossRef] [PubMed]

15. Scozzafava, A.; Passaponti, M.; Supuran, C.T.; Gülçin, İ. Carbonic anhydrase inhibitors: Guaiacol and catechol derivatives effectively inhibit certain human carbonic anhydrase isoenzymes (hCA I, II, IX, and XII). J. Enzym. Inhib. Med. Chem. 2015, 30, 586-591. [CrossRef] [PubMed]

16. Göçer, H.; Akıncıoğlu, A.; Göksu, S.; Gülçin, İ.; Supuran, C.T. Carbonic anhydrase and acetylcholine esterase inhibitory effects of carbamates and sulfamoylcarbamates. J. Enzym. Inhib. Med. Chem. 2015, 30, 316-320. [CrossRef] [PubMed]

17. Arabaci, B.; Gülçin, İ.; Alwasel, S. Capsaicin: A potent inhibitor of carbonic anhydrase isoenzymes. Molecules 2014, 19, 10103-10114. [CrossRef] [PubMed]

18. Akbaba, Y.; Bastem, E.; Topal, F.; Gülçin, İ.; Maraş, A.; Göksu, S. Synthesis and carbonic anhydrase inhibitory effects of novel sulfamides derived from 1-aminoindanes and anilines. Arch. Pharm. 2014, 347, 950-957. [CrossRef] [PubMed]

19. Göksu, S.; Naderi, A.; Akbaba, Y.; Kalın, P.; Akıncıoğlu, A.; Gulcin, İ.; Durdaği, S.; Salmas, R.E. Carbonic anhydrase inhibitory properties of novel benzylsulfamides using molecular modeling and experimental studies. Bioorg. Chem. 2014, 56, 75-82. [CrossRef] [PubMed]

20. Fujikawa-Adachi, K.; Nishimori, I.; Taguchi, T.; Onishi, S. Human mitochondrial carbonic anhydrase VB. cDNA cloning, mRNA expression, subcellular localization, and mapping to chromosome x. J. Biol. Chem. 1999, 274, 21228-21233. [CrossRef] [PubMed]

21. Nagao, Y.; Platero, J.S.; Waheed, A.; Sly, W.S. Human mitochondrial carbonic-anhydrase-cDNA cloning, expression, subcellular-localization, and mapping to chromosome-16. Am. J. Hum. Genet. 1993, 53, 7623-7627. [CrossRef]

22. Topal, M.; Gülçin, I. Rosmarinic acid: a potent carbonic anhydrase isoenzymes inhibitor. Turk. J. Chem. 2014, 38, 894-902. [CrossRef]

23. Çetinkaya, Y.; Göçer, H.; Gülçin, I.; Menzek, A. Synthesis and carbonic anhydrase isoenzymes inhibitory effects of brominated diphenylmethanone and its derivatives. Arch. Pharm. 2014, 347, 354-359. [CrossRef] [PubMed]

24. Çetinkaya, Y.; Göçer, H.; Göksu, S.; Gülçin, I. Synthesis and carbonic anhydrase isoenzymes inhibitory effects of novel benzylamine derivatives. J. Enzym. Inhib. Med. Chem. 2014, 29, 168-174. [CrossRef] [PubMed]

25. Akbaba, Y.; Akıncıoğlu, A.; Göçer, H.; Göksu, S.; Gülçin, I.; Supuran, C.T. Carbonic anhydrase inhibitory properties of novel sulfonamide derivatives of aminoindanes and aminotetralins. J. Enzym. Inhib. Med. Chem. 2014, 29, 35-42. [CrossRef] [PubMed]

26. Özgeriş, B.; Göksu, S.; Köse Polat, L.; Gülçin, I.; Salmas, R.E.; Durdagi, S.; Tümer, F.; Supuran, C.T. Acetylcholinesterase and carbonic anhydrase inhibitory properties of novel urea and sulfamide derivatives incorporating dopaminergic 2-aminotetralin scaffolds. Bioorg. Med. Chem. 2016, 24, 2318-2329. [CrossRef] [PubMed]

27. Aksu, K.; Nar, M.; Tanç, M.; Vullo, D.; Gülçin, I.; Göksu, S.; Tümer, F.; Supuran, C.T. The synthesis of sulfamide analogues of dopamine related compounds and their carbonic anhydrase inhibitory properties. Bioorg. Med. Chem. 2013, 21, 2925-2931. [CrossRef] [PubMed]

28. Akıncıoğlu, A.; Akbaba, Y.; Göçer, H.; Göksu, S.; Gülçin, I.; Supuran, C.T. Novel sulfamides as potential carbonic anhydrase isoenzymes inhibitors. Bioorg. Med. Chem. 2013, 21, 1379-1385. [CrossRef] [PubMed]

29. Gülçin, I.; Beydemir, S. Phenolic compounds as antioxidants: Carbonic anhydrase isoenzymes inhibitors. Mini Rev. Med. Chem. 2013, 13, 408-430. [PubMed]

30. Nar, M.; Çetinkaya, Y.; Gülçin, I.; Menzek, A. (3,4-Dihydroxyphenyl) (2,3,4-trihydroxyphenyl) methanone and its derivatives as carbonic anhydrase isoenzymes inhibitors. J. Enzym. Inhib. Med. Chem. 2013, 28, 402-406. [CrossRef] [PubMed]

31. Öztürk Sarıkaya, S.B.; Topal, F.; Şentürk, M.; Gülçin, I.; Supuran, C.T. In vitro inhibition of $\alpha$-carbonic anhydrase isozymes by some phenolic compounds. Bioorg. Med. Chem. Lett. 2011, 21, 4259-4262. [CrossRef] [PubMed]

32. Şentürk, M.; Gülçin, I.; Beydemir, Ş.; Küfrevioğlu, Ö.I.; Supuran, C.T. In vitro inhibition of human carbonic anhydrase I and II isozymes with natural phenolic compounds. Chem. Biol. Drug Des. 2011, 77, 494-499. [CrossRef] [PubMed] 
33. Lomelino, C.L.; Supuran, C.T.; McKenna, R. Non-classical inhibition of carbonic anhydrase. Int. J. Mol. Sci. 2016, 17, 1150. [CrossRef] [PubMed]

34. Innocenti, A.; Gülçin, I.; Scozzafava, A.; Supuran, C.T. Carbonic anhydrase inhibitors. Antioxidant polyphenol natural products effectively inhibit mammalian isoforms I-XV. Bioorg. Med. Chem. Lett. 2010, 20, 5050-5053. [CrossRef] [PubMed]

35. Innocenti, A.; Öztürk Sarıkaya, S.B.; Gülçin, I.; Supuran, C.T. Carbonic anhydrase inhibitors. Inhibition of mammalian isoforms I-XIV with a series of natural product polyphenols and phenolic acids. Bioorg. Med. Chem. 2010, 18, 2159-2164. [CrossRef] [PubMed]

36. Polat Köse, L.; Gülçin, I.; Gören, A.C.; Namiesnik, J.; Martinez-Ayala, A.L.; Gorinstein, S. LC-MS/MS analysis, antioxidant and anticholinergic properties of galanga (Alpinia officinarum Hance) rhizomes. Ind. Crops Prod. 2015, 74, 712-721. [CrossRef]

37. Aksu, K.; Topal, F.; Gülçin, I.; Tümer, F.; Göksu, S. Acetylcholinesterase inhibitory and antioxidant activities of novel symmetric sulfamides derived from phenethylamines. Arch. Pharm. 2015, 348, 446-455. [CrossRef] [PubMed]

38. Akıncıoğlu, A.; Topal, M.; Gülçin, I.; Göksu, S. Novel sulfamides and sulfonamides incorporating tetralin scaffold as carbonic anhydrase and acetylcholine esterase inhibitors. Arch. Pharm. 2014, 347, 68-76. [CrossRef] [PubMed]

39. Göçer, H.; Akıncıoğlu, A.; Öztaşkın, N.; Göksu, S.; Gülçin, I. Synthesis, antioxidant and antiacetylcholinesterase activities of sulfonamide derivatives of dopamine related compounds. Arch. Pharm. 2013, 346, 783-792. [CrossRef] [PubMed]

40. Gocer, H.; Topal, F.; Topal, M.; Küçük, M.; Teke, D.; Gulcin, I.; Alwasel, S.H.; Supuran, C.T. Acetylcholinesterase and carbonic anhydrase isoenzymes I and II inhibition profiles of taxifolin. J. Enzym. Inhib. Med. Chem. 2016, 31, 441-447. [CrossRef] [PubMed]

41. Van der Zee, E.A.; Platt, B.; Riedel, G. Localization of pre- and postsynaptic cholinergic markers in rodent forebrain: A brief history and comparison of rat and mouse. Behav. Brain Res. 2011, 221, 583-586. [CrossRef] [PubMed]

42. Achilles, K.; Schirmeister, T.; Otto, H.H. $\beta$-Lactam derivatives as enzyme inhibitors: 1-Peptidyl derivatives of 4-phenylazetidin-2-one as inhibitors of elastase and papain. Arch. Pharm. 2000, 333, 243-253. [CrossRef]

43. Mollet, K. Transformation of trans-4-aryl-3-chloro-1-(2-chloroethyl)azetidin-2-ones into 3-aryl-2(ethylamino)propan-1-ols via intermediate 1-(1-aryl-2-chloro-3-hydroxypropyl)aziridines and trans-2-aryl-3(hydroxymethyl)aziridines. J. Org. Chem. 2010, 76, 264-269. [CrossRef] [PubMed]

44. Zhao, L. Functionalization of the $\mathrm{C}-\mathrm{H}$ Bond Adjacent to a Secondary Nitrogen Atom. Ph.D. Thesis, McGill University, Montréal, QC, Canada, August 2009.

45. France, S.; Shah, M.H.; Weatherwax, A.; Wack, H.; Roth, J.P.; Lectka, T. Bifunctional Lewis acid-nucleophile-based asymmetric catalysis: Mechanistic evidence for imine activation working in tandem with chiral enolate formation in the synthesis of $\beta$-lactams. J. Am. Chem. Soc. 2005, 127, 1206-1215. [CrossRef] [PubMed]

46. Shapiro, R.; Vallee, B.L. Interaction of human placental ribonuclease with placental ribonuclease inhibitor. Biochemistry 1991, 30, 2246-2255. [CrossRef] [PubMed]

47. Supuran, C.T. Carbonic anhydrase inhibitors. Bioorg. Med. Chem. Lett. 2010, 20, 3467-3474. [CrossRef] [PubMed]

48. Gülçin, I.; Beydemir, Ş.; Büyükokuroglu, M.E. In vitro and in vivo effects of dantrolene on carbonic anhydrase enzyme activities. Biol. Pharm. Bull. 2004, 27, 613-616. [CrossRef] [PubMed]

49. Innocenti, A.; Maresca, A.; Scozzafava, A.; Supuran, C.T. Carbonic anhydrase inhibitors: thioxolone versus sulfonamides for obtaining isozyme-selective inhibitors? Bioorg. Med. Chem. Lett. 2008, 18, 3938-3941. [CrossRef] [PubMed]

50. Hochster, R.M.; Kates, M.; Quaste, J.H. Metabolic Inhibitors: A Comprehensive Treatise; Academic Press: New York, NY, USA, 1973; Volume 4, pp. 66-82.

51. Christensen, G.M.; Olson, D.; Riedel, B. Chemical effects on the activity of eight enzymes: A review and discussion relevant to environmental monitoring. Environ. Res. 1982, 29, 247-255. [CrossRef]

52. Turan, B.; Sendil, K.; Sengul, E.; Gultekin, M.S.; Taslimi, P.; Gulcin, I.; Supuran, C.T. The synthesis of some $\beta$-lactams and investigation of their metal chelating activity, carbonic anhydrase and achetylcholinesterase inhibition profiles. J. Enzym. Inhib. Med. Chem. 2016. [CrossRef] [PubMed] 
53. Berber, N.; Arslan, M.; Bilen, C.; Sackes, Z.; Gencer, N.; Arslan, O. Synthesis and evaluation of new phthalazine substituted $\beta$-lactam derivatives as carbonic anhydrase inhibitors. Russ. J. Bioorg. Chem. 2015, 41, 414-420. [CrossRef]

54. Şişecioğlu, M.; Gülçin, I.; Çankaya, M.; Özdemir, H. The inhibitory effects of L-Adrenaline on lactoperoxidase enzyme (LPO) purified from buffalo milk. Int. J. Food Propert. 2012, 15, 1182-1189. [CrossRef]

55. Polat Kose, L.; Gülçin, I.; Özdemir, H.; Atasever, A.; Alwasel, S.H.; Supuran, C.T. The effects of some avermectins on bovine carbonic anhydrase enzyme. J. Enzym. Inhib. Med. Chem. 2016, 31, 773-778. [CrossRef] [PubMed]

56. Lineweaver, H.; Burk, D. The determination of enzyme dissociation constants. J. Am. Chem. Soc. 1934, 56, 658-666. [CrossRef]

57. Atasever, A.; Özdemir, H.; Gülçin, I.; Küfrevioğlu, Ö.İ. One-step purification of lactoperoxidase from bovine milk by affinity chromatography. Food Chem. 2013, 136, 864-870. [CrossRef] [PubMed]

58. Öztürk Sarıkaya, S.B.; Gülçin, I.; Supuran, C.T. Carbonic anhydrase inhibitors. Inhibition of human erythrocyte isozymes I and II with a series of phenolic acids. Chem. Biol. Drug Des. 2010, 75, 515-520. [CrossRef] [PubMed]

59. Şentürk, M.; Gülçin, I.; Daştan, A.; Küfrevioğlu, Ö.İ.; Supuran, C.T. Carbonic anhydrase inhibitors. Inhibition of human erythrocyte isozymes I and II with a series of antioxidant phenols. Bioorg. Med. Chem. 2009, 17, 3207-3211. [CrossRef] [PubMed]

60. Çoban, T.A.; Beydemir, Ş.; Gülçin, I.; Ekinci, D. The inhibitory effect of ethanol on carbonic anhydrase isoenzymes: In vivo and in vitro studies. J. Enzym. Inhib. Med. Chem. 2008, 23, 266-270. [CrossRef] [PubMed]

61. Çoban, T.A.; Beydemir, Ş.; Gülçin, I.; Ekinci, D. Morphine inhibits erythrocyte carbonic anhydrase in vitro and in vivo. Biol. Pharm. Bull. 2007, 30, 2257-2261. [CrossRef] [PubMed]

62. Hisar, O.; Beydemir, Ş.; Gülçin, I.; ArasHisar, Ş.; Yanık, T.; Küfrevioğlu, Ö.İ. The effect of melatonin hormone on carbonic anhydrase enzyme activity in rainbow trout (Oncorhynchus mykiss) erythrocytes in vitro and in vivo. Turk. J. Vet. Anim. Sci. 2005, 29, 841-845.

63. Bradford, M.M. A rapid and sensitive method for the quantitation of microgram quantities of protein utilizing the principle of protein-dye binding. Anal. Biochem. 1976, 72, 248-254. [CrossRef]

64. Gülçin, I.; Küfrevioğlu, Ö.İ.; Oktay, M. Purification and characterization of polyphenol oxidase from nettle (Urtica dioica L.) and inhibition effects of some chemicals on the enzyme activity. J. Enzym. Inhib. Med. Chem. 2005, 20, 297-302. [CrossRef] [PubMed]

65. Köksal, E.; Gülçin, İ. Purification and characterization of peroxidase from cauliflower (Brassica oleracea L.) buds. Protein Pept. Lett. 2008, 15, 320-326. [CrossRef] [PubMed]

66. Şentürk, M.; Gülçin, I.; Çiftci, M.; Küfrevioğlu, Ö.İ. Dantrolene inhibits human erythrocyte glutathione reductase. Biol. Pharmacol. Bull. 2008, 31, 2036-2039. [CrossRef]

67. Laemmli, D.K. Cleavage of structural proteins during the assembly of the head of bacteriophage T4. Nature 1970, 227, 680-683. [CrossRef] [PubMed]

68. Gülçin, I.; Beydemir, Ş.; Çoban, T.A.; Ekinci, D. The inhibitory effect of dantrolene sodium and propofol on 6-phosphogluconate dehydrogenase from rat erythrocyte. Fresenius Environ. Bull. 2008, 17, 1283-1287.

69. Şişecioğlu, M.; Çankaya, M.; Gülçin, I.; Özdemir, M. The Inhibitory effect of propofol on lactoperoxidase. Protein Pept. Lett. 2009, 16, 46-49. [CrossRef] [PubMed]

70. Şişecioğlu, M.; Çankaya, M.; Gülçin, I.; Özdemir, H. Interactions of melatonin and serotonin to lactoperoxidase enzyme. J. Enzym. Inhib. Med. Chem. 2010, 25, 779-783. [CrossRef] [PubMed]

71. Verpoorte, J.A.; Mehta, S.; Edsall, J.T. Esterase activities of human carbonic anhydrases B and C. J. Biol. Chem. 1967, 242, 4221-4229. [PubMed]

72. Şişecioğlu, M.; Gülçin, I.; Çankaya, M.; Atasever, A.; Şehitoğlu, M.H.; Budak Kaya, H.; Özdemir, H. Purification and characterization of peroxidase from Turkish black radish (Raphanus sativus L.). J. Med. Plants Res. 2010, 4, 1187-1196.

73. Köksal, E.; Ağgül, A.G.; Bursal, E.; Gülçin, I. Purification and characterization of peroxidase from sweet gourd (Cucurbita Moschata Lam. Poiret). Int. J. Food Propert. 2012, 15, 1110-1119. [CrossRef]

74. Şişecioğlu, M.; Kireçci, E.; Çankaya, M.; Özdemir, H.; Gülçin, I.; Atasever, A. The prohibitive effect of lactoperoxidase system (LPS) on some pathogen fungi and bacteria. Afr. J. Pharm. Pharmacol. 2010, 4, 671-677. 
75. Ellman, G.L.; Courtney, K.D.; Andres, V.; Featherston, R.M. A new and rapid colorimetric determination of acetylcholinesterase activity. Biochem. Pharmacol. 1961, 7, 88-95. [CrossRef]

76. Gül, H.İ.; Kucukoglu, K.; Yamali, C.; Bilginer, S.; Yuca, H.; Ozturk, İ.; Taslimi, P.; Gülçin, I.; Supuran, C.T. Synthesis of 4-(2-substitutedhydrazinyl)benzenesulfonamides and their carbonic anhydrase inhibitory effects. J. Enzym. Inhib. Med. Chem. 2016, 31, 568-573.

77. Goksu, H.; Topal, M.; Keskin, A.; Gultekin, M.S.; Çelik, M.; Gülçin, I.; Tanc, M.; Supuran, C.T. 9,10-Dibromo- $N$-aryl-9,10-dihydro-9,10-[3,4] epipyrroloanthracene-12,14-diones: Synthesis and investigation of their effects on carbonic anhydrase isozymes I, II, IX, and XII. Arch. Pharm. 2016, 349, 466-474. [CrossRef] [PubMed]

(C) 2016 by the authors; licensee MDPI, Basel, Switzerland. This article is an open access article distributed under the terms and conditions of the Creative Commons Attribution (CC-BY) license (http:/ / creativecommons.org/licenses/by/4.0/). 